\title{
Optimal Estimation on the Graph Cycle Space
}

\author{
Wm. Joshua Russell Daniel J. Klein João P. Hespanha
}

\begin{abstract}
This paper addresses the problem of estimating the states of a group of agents from noisy measurements of pairwise differences between agents' states. The agents can be viewed as nodes in a graph and the relative measurements between agents as the graph's edges. We propose a new distributed algorithm that exploits the existence of cycles in the graph to compute the best linear state estimates.

For large graphs, the new algorithm significantly reduces the total number of message exchanges that are needed to obtain an optimal estimate. We show that the new algorithm is guaranteed to converge for planar graphs and provide explicit formulas for its converge rate for regular lattices.
\end{abstract}

\section{INTRODUCTION}

As large scale sensor networks have become more prevalent in military and consumer applications, increasing interest has been paid to distributed estimation and its applications. Common motivations for distributed estimation include localization, target tracking, and sensor fusion applications. The goal of these problems is to determine, without knowledge of the whole network, a best estimate of some state within the network from an over constrained set of measurements.

There have been many approaches to the distributed estimation problem for different applications. Among the simplest distributed estimation problems is the linear consensus problem addressed in [4]. More challenging problems such as distributed estimation of clock skew and offset have been explored in [1] and [9]. More challenging still, are problems such as track-to-track fusion [2] and localization from time differences of arrival [5].

One approach to distributed estimation that is particularly inspiring to this paper came from Barooah et al. In [1], Barooah examines distributed estimation from relative difference measurements. The relative difference measurement problem assumes that each node in a network has an associated state that cannot be measured absolutely (e.g. a node's global position), but a node can measure the relative difference between its state and its neighbor's states (e.g. a node can measure the relative position of its neighbors). Barooah has explored several distributed algorithms for this problem that converge to the optimal minimum error variance solution. Barooah's algorithms assume that nodes have very limited topological knowledge and communication abilities. In these algorithms, each node updates an estimate of its own

This material is based upon work supported by the Institute for Collaborative Biotechnologies through grant DAAD19-03-D-0004 from the U.S. Army Research Office and by the National Science Foundation under Grant No. CNS-0720842.

All authors are with the Department of Electrical and Computer Engineering, University of California, Santa Barbara, CA 93106. \{wjrussell, djklein, hespanha\}eece.ucsb.edu state synchronously using an affine transformation of neighboring states. Methods of this sort estimate the node state directly and are referred to as node estimation methods. The node estimation methods introduced in [1] always converge to the optimal estimate and have been shown to be robust to interruptions in communication.

This work is motivated by the observation that, in the absence of noise, the sum of the relative difference measurements along any cycle of the graph should be zero. However, due to measurement noise, these sums are generally not equal to zero - in this paper we refer to these sums as discrepancies. The Cycle Space Estimation algorithms introduced in this paper consist of two steps: first, one computes a "corrected" version of the measurement set that has zero discrepancy and then one uses this corrected measurement set to estimate the state variables. The first step can be done either in a centralized or distributed fashion. Its decentralized version utilizes a Jacobi-like algorithm [7] on a graph whose nodes correspond to the faces of the original graph. The second step can also be done in a centralized or distributed fashion. In the latter case, convergence is guaranteed within a finite number of steps (equal to the graph diameter) using the so-called flagged initialization algorithm [1].

For large classes of graphs, the new distributed algorithms converge significantly faster than previous algorithms. This happens because the "faces graph" often has much better convergence properties than the original graph. We show through Monte Carlo simulations that this is the case for several classes of regular graphs, which include square, triangular, and hexagonal lattices of varying size. For square lattices, we are also able to determine explicit formulas to compute the converge rate of our distributed algorithms. Overall our results show that, for large graphs, one can obtain significant gains in terms of the number of messages that are required to compute optimal estimates.

This paper proceeds in the following manner. Section II provides a review of the problem statement and optimal estimation discussed in [1]. In Section III, centralized cycle space estimation is introduced. Distributed cycle space estimation is covered in Section IV. The convergence rates of distributed cycle space estimation are discussed in Section V. Simulation results are given in Section VI. Conclusions and future research goals are discussed in Section VII.

\section{Centralized Node Estimation}

Consider a network of sensors, each with a vector-valued node state in $d$-dimensions. The sensors take relative measurements, which consist of the difference between the 
states of two sensors. The measurements are corrupted by uncorrelated zero-mean Gaussian noise with (possibly) nonuniform covariances. The problem we are interested in is to estimate each sensor's state in an optimal fashion.

Let $\mathcal{G}=(\mathcal{V}, \mathcal{E})$ be the measurement directed graph associated with the estimation problem. The node set, $\mathcal{V}$, is the set of sensors. The edge set, $\mathcal{E}$, contains all pairs of sensors for which there is a relative measurement. By convention, the direction of the edge goes from the node that appears with the plus sign in the relative difference to the node that appears with the minus sign. Let $n$ and $m$ be the cardinality of $\mathcal{V}$ and the cardinality of $\mathcal{E}$ respectively. The measurement graph can be represented compactly by its incidence matrix $\bar{B}=\bar{b}_{i j}, i \in(1,2, \ldots, n), j \in(1,2, \ldots, m)$,

$$
\bar{b}_{i j} \equiv \begin{cases}1 & \text { if edge } \mathrm{j} \text { leaves node } \mathrm{i} \\ -1 & \text { if edge } \mathrm{j} \text { enters node } \mathrm{i} \\ 0 & \text { otherwise }\end{cases}
$$

Let $B$ be the Kronecker product of $\bar{B}$ and an identity matrix of size $d$. Let $\tilde{z}$ be the vector of stacked measurements, let $\varepsilon$ be the vector of measurement noise, and let $x_{g}$ be the vector of states of elements in $\mathcal{V}$ (note that $\tilde{z}$ and $\varepsilon$ are length $d m$ and $x_{g}$ is length $d n$ ). Then $x_{g}, \tilde{z}$, and $\varepsilon$ are related by:

$$
\tilde{z}=B^{\prime} x_{g}+\varepsilon
$$

Because measurements are taken as relative differences in state, any solution, $\hat{x_{g}}$, to (1) is unique only up to an additive constant. To remove this degree of freedom, a subset of the nodes are taken as references and it is assumed that the states of these nodes are known. Often a single reference node is used and its state is assumed zero, but here we consider the more general case of multiple references. The whole state, $x_{g}$, can be split into two vectors $x_{r}$ and $x$ containing, respectively, the states of the references nodes and the states of the nodes that need to be estimated. Likewise, $B$ can be split into two matrices, $B_{r}$ and $B_{e}$ containing, respectively, the rows of $B$ corresponding to reference nodes and the rows of $B$ corresponding to nodes where the state is to be estimated. Moving the reference states (and corresponding columns of $B^{\prime}$ ) to the left-hand side of (1), we obtain:

$$
\tilde{z}-B_{r}^{\prime} x_{r}=B_{e}^{\prime} x+\varepsilon .
$$

Lastly, define the measurement covariance matrix as $P \equiv$ $E\left[\varepsilon \varepsilon^{\prime}\right] ; P$ is a symmetric non-singular block diagonal matrix. It was shown in [1] that given a weakly connected (i.e. disregarding edge direction, the graph is connected) measurement graph, the Best Linear Unbiased Estimator (BLUE) for the non-reference states $x$ given the measurements $\tilde{z}$ is:

$$
\hat{x}^{*}=\left(B_{e} P^{-1} B_{e}^{\prime}\right)^{-1} B_{e} P^{-1}\left(\tilde{z}-B_{r}^{\prime} x_{r}\right) .
$$

In other words, (2) gives the centralized state estimate with minimum variance.

\section{Centralized Optimal Cycle Space Estimation}

To gain insight into the approach followed here, it is convenient to regard the states of the nodes as voltages in an electric circuit that takes the shape of the measurement graph. The measurements would then correspond to tensions measured across the edges of the graph. When the graph has cycles, one should obtain zero by summing the tensions along a cycle, but because of measurement errors this is generally not the case. In this light, one can view the estimation procedure as computing a set of voltages that are internally consistent, in the sense that they add up to zero around any cycle.

Let us define the vector of optimal tension estimates as:

$$
\hat{z}^{*} \equiv\left[\begin{array}{ll}
B_{r}^{\prime} & B_{e}^{\prime}
\end{array}\right]\left[\begin{array}{l}
x_{r} \\
\hat{x}^{*}
\end{array}\right],
$$

where $\hat{x}^{*}$ is the minimum variance estimate for $x$ from (2). The vector $\hat{z}^{*}$ defined above is obtained by taking differences between all elements of the optimal estimate $\hat{x}^{*}$ for which we have measurements available. In the absence of noise, $\hat{x}^{*}$ would be equal to $x$ and $\hat{z}^{*}$ would be equal to the measurement vector, $\tilde{z}$.

While it appears from (3) that we first need to compute the optimal estimate $\hat{x}^{*}$ to compute the optimal tension estimate $\hat{z}^{*}$, we will see shortly that it is possible to compute $\hat{z}^{*}$ directly from the measurements $\tilde{z}$ and that it is then straightforward to obtain $\hat{x}^{*}$ from $\hat{z}^{*}$. We shall also see that this indirect procedure can be advantageous from the perspective of reducing computation.

\section{A. Cycle Space}

A simple cycle of $\mathcal{G}$ is a directed path in $\mathcal{G}$ that starts and ends at the same node and has only this one repeated node. By indexing the edges with natural numbers 1 through $m$, we can associate to each simple cycle a simple cycle vector, which is defined to be the vector $c=c_{i}, i \in(1,2, \ldots, m)$,

$$
c_{i} \equiv \begin{cases}1 & \text { if cycle traverses edge i forward } \\ -1 & \text { if cycle traverses edge i backward } \\ 0 & \text { otherwise. }\end{cases}
$$

The cycle space, $\mathcal{C}$, of $\mathcal{G}$ is then defined to be the subspace of $\mathbb{R}^{m}$ generated by the simple cycle vectors associated with the simple cycles in $\mathcal{G}$. Subsequently, $C$ will be used to denote a matrix whose columns are simple cycle vectors that form a basis for the cycle space. Considering that every column of $B$ corresponds to an edge in $\mathcal{G}, C$ is a basis for $\operatorname{null}(B)$ [8]. The cycle space matrix, $C$, has height $m$ and width $r$, where $r$ is the dimension of $\mathcal{C}$.

The Centralized Optimal Cycle Space Estimation (COCSE) algorithm uses $\tilde{z}$ and $C$ to compute $\hat{z}^{*}$. The premise of the COCSE algorithm is to add a correction term to the measurements, $\tilde{z}$, to form the optimal set of tensions, $\hat{z}^{*}$. 


\section{B. The Centralized Optimal Cycle Space Estimate}

Theorem 1: [Optimality of Cycle Space Estimation]

The vector of optimal tension estimates defined by (3) is related to the measurement vector in (1) by the following formula:

$$
\hat{z}^{*} \equiv \begin{cases}\tilde{z} & \text { if } r=0 \\ \tilde{z}-P C\left(C^{\prime} P C\right)^{-1} C^{\prime} \tilde{z} & \text { if } r \neq 0 .\end{cases}
$$

Proof: Lemma 1 (in the Appendix) is used extensively in this proof. This lemma gives a relation for the projections of two spaces that are orthogonal complements. Namely, if subspaces $\alpha^{\prime}$ and $\beta$ are orthogonal complements, then $\operatorname{proj}\left(\alpha^{\prime}\right)=I-\operatorname{proj}(\beta)$.

If $r=0$ ( $\mathcal{G}$ has no cycles) then the graph, $\mathcal{G}$, is a tree. Consequentially, the set of measurements are consistent and represent the BLUE.

To prove (4) when $r \neq 0$, consider the linear subspaces $\beta=\operatorname{image}\left(B_{e} P^{-\frac{1}{2}}\right)$ and $\alpha=\operatorname{image}\left(P^{\frac{1}{2}} C\right)$. Note that $\operatorname{null}(\operatorname{proj}(\alpha))=\beta$. Considering Lemma 1 and the fact that $C^{\prime} B_{r}^{\prime}=0$, the following equalities hold:

$$
\begin{aligned}
0 & =\left[\alpha\left(\alpha^{\prime} \alpha\right)^{-1} C^{\prime} P^{\frac{1}{2}}\right] P^{-\frac{1}{2}} B_{r}^{\prime} x_{r} \\
& =\left[\alpha\left(\alpha^{\prime} \alpha\right)^{-1} \alpha^{\prime}\right] P^{-\frac{1}{2}} B_{r}^{\prime} x_{r} \\
& =\left[I-\beta^{\prime}\left(\beta \beta^{\prime}\right)^{-1} \beta\right] P^{-\frac{1}{2}} B_{r}^{\prime} x_{r} \\
& =P^{\frac{1}{2}}\left[I-\beta^{\prime}\left(\beta \beta^{\prime}\right)^{-1} \beta\right] P^{-\frac{1}{2}} B_{r}^{\prime} x_{r} \\
& =\left[I-P^{\frac{1}{2}} \beta^{\prime}\left(\beta \beta^{\prime}\right)^{-1} \beta P^{-\frac{1}{2}}\right] B_{r}^{\prime} x_{r} \\
& =\left[I-B_{e}^{\prime}\left(B_{e} P^{-1} B_{e}^{\prime}\right)^{-1} B_{e} P^{-1}\right] B_{r}^{\prime} x_{r} .
\end{aligned}
$$

Consider again Lemma 1:

$$
\begin{aligned}
I-\alpha\left(\alpha^{\prime} \alpha\right)^{-1} \alpha^{\prime} & =\beta^{\prime}\left(\beta \beta^{\prime}\right)^{-1} \beta \\
I-P^{\frac{1}{2}} \alpha\left(\alpha^{\prime} \alpha\right)^{-1} \alpha^{\prime} P^{-\frac{1}{2}} & =P^{\frac{1}{2}} \beta^{\prime}\left(\beta \beta^{\prime}\right)^{-1} \beta P^{-\frac{1}{2}} \\
I-P C\left(C^{\prime} P C\right)^{-1} C^{\prime} & =B_{e}^{\prime}\left(B_{e} P^{-1} B_{e}^{\prime}\right)^{-1} B_{e} P^{-1} \\
{\left[I-P C\left(C^{\prime} P C\right)^{-1} C^{\prime}\right] \tilde{z} } & =B_{e}^{\prime}\left(B_{e} P^{-1} B_{e}^{\prime}\right)^{-1} B_{e} P^{-1} \tilde{z} .
\end{aligned}
$$

Now, add (5) to (6):

$$
\begin{gathered}
{\left[I-P C\left(C^{\prime} P C\right)^{-1} C^{\prime}\right] \tilde{z}=B_{e}^{\prime}\left(B_{e} P^{-1} B_{e}^{\prime}\right)^{-1} B_{e} P^{-1} \tilde{z}+} \\
{\left[I-B_{e}^{\prime}\left(B_{e} P^{-1} B_{e}^{\prime}\right)^{-1} B_{e} P^{-1}\right] B_{r}^{\prime} x_{r}} \\
=B_{r}^{\prime} x_{r}+ \\
B_{e}^{\prime}\left(B_{e} P^{-1} B_{e}^{\prime}\right)^{-1} B_{e} P^{-1}\left(\tilde{z}-B_{r}^{\prime} x_{r}\right) \\
=B_{r}^{\prime} x_{r}+B_{e}^{\prime} \hat{x}^{*} \equiv \hat{z}^{*} .
\end{gathered}
$$

\section{Physical Interpretation of the Optimal Tension Estimate}

The formal definition of $\hat{z}^{*}$ in (3) sheds little light into why it is useful to consider such quantity. The following result shows that the optimal tension estimates vector, $\hat{z}^{*}$, can be viewed as a least-squares approximation to the measurement vector $\tilde{z}$ subject to the constraint that it must belong to the kernel of the cycle space basis matrix $C$.
Theorem 2: [Quadratic Programming Solution of $\hat{z}$ ] Equation (4) is the solution to the quadratic programming problem:

$$
\min _{\hat{z}}\left[(\tilde{z}-\hat{z})^{\prime} P^{-1}(\tilde{z}-\hat{z})\right] \quad \text { s.t. } C^{\prime} \hat{z}=0 .
$$

Proof: When $r=0$ ( $\mathcal{G}$ has no cycles), $C^{\prime}$ is trivial and the constraint in (7) is satisfied for any $\hat{z}$. Consequentially, $\tilde{z}^{*}=\hat{z}$ as in equation (4).

When $r \neq 0$, the Lagrangian for (7) is:

$$
L(\hat{z}, \lambda)=(\tilde{z}-\hat{z})^{\prime} P^{-1}(\tilde{z}-\hat{z})+\lambda^{\prime} C^{\prime} \hat{z} .
$$

Taking the partial derivatives of the Lagrangian equal to zero:

$$
\begin{aligned}
& \frac{\partial L}{\partial \hat{z}}=2 P^{-1}(\hat{z}-\tilde{z})+C \lambda=0 \\
& \frac{\partial L}{\partial \lambda}=C^{\prime} \hat{z}=0,
\end{aligned}
$$

which can be written in the form:

$$
\left[\begin{array}{cc}
2 P^{-1} & C \\
C^{\prime} & 0
\end{array}\right]\left[\begin{array}{c}
\hat{z} \\
\lambda
\end{array}\right]=\left[\begin{array}{c}
2 P^{-1} \tilde{z} \\
0
\end{array}\right]
$$

Using the Schur Complement, (8) can be solved to find $\hat{z}^{*}$ :

$$
\begin{aligned}
\hat{z}^{*} & =\left[\frac{1}{2} P-\frac{1}{2} P C^{\prime}\left(C P C^{\prime}\right)^{-1} C P\right] 2 P^{-1} \tilde{z} \\
& =\left[I-P C^{\prime}\left(C P C^{\prime}\right)^{-1} C\right] \tilde{z} .
\end{aligned}
$$

Noting that (9) is equivalent to (4), the following interpretation of $\hat{z}^{*}$ can be made: The set of tensions corresponding to the BLUE, $\hat{z}^{*}$, is the set of tensions with the least square difference from the set of measurements, $\tilde{z}$. That is to say, it is the set of tensions providing the minimum squared edge modification.

\section{Cycle Laplacian}

Stemming from the derivation of the COCSE is the cycle Laplacian matrix,

$$
\mathcal{L}_{c} \equiv C^{\prime} P C .
$$

While $\mathcal{L}_{c}$ is generally not doubly stochastic, we call it a Laplacian because it characterizes the weighted cycle degree and weighted cycle adjacency, which are defined as follows: let $\gamma_{i} \subset \mathcal{E}$ be the set of edges in the cycle corresponding to the $i^{\text {th }}$ column of the cycle space basis matrix $C$. The weighted cycle degree, $D_{i}$, of this cycle is the sum of the error covariance matrices that correspond to the measurements in cycle $i$, i.e., $D_{i}=\sum_{\ell=\gamma_{i}} P_{\ell}$. If two cycles, $i, j \subset \mathcal{C}$, traverse their common edges (i.e. edges that are in both cycles) in the same direction, then the weighted cycle adjacency between the cycles, $A_{i j}$, is the sum of the covariance matrices corresponding to the edges common to both cycles, i.e., $A_{i j}=\sum_{\ell=\gamma_{i \cap j}} P_{\ell}$. If two cycles, $i, j \subset \mathcal{C}$, traverse their common edges in the opposite direction, then the weighted adjacency between the cycles, $A_{i j}$, is the product of negative one and the sum of the covariance matrices corresponding to the edges 
common to both cycles, i.e., $A_{i j}=-\sum_{\ell=\gamma_{i \cap j}} P_{\ell}$. If two cycles, $i, j \subset \mathcal{C}$, have no common edges, then the weighted adjacency between the cycles, $A_{i j}$, is zero. The weighted cycle degree and weighted cycle adjacency are related to the cycle Laplacian by $\mathcal{L}_{c}=D-A ; D=\operatorname{diag}\left(D_{1}, \ldots, D_{o}\right)$ and $A=\left[A_{i j}\right] . \mathcal{L}_{c}$ has size $o d$, where $o$ is the rank of $C$.

\section{Distributed Estimation in the Cycle Space}

In a large network it is often impractical to collect all of the measurements at one node, compute the optimal estimate at this node, and then redistribute the estimate to every node. Instead, it is desirable to find an estimate of the state in a distributed manner. To construct such a distributed algorithm, we make the following assumptions:

1) A node knows the measurements corresponding its edges

2) A basis $C$ for the cycle space of the graph is known and a "leader" has been elected for each simple cycle corresponding to the columns of $C$

3) Each node knows how to send messages to each one of its neighbors in $\mathcal{G}$

4) All nodes that belong to one (or more) of the simple cycles associated with a column of $C$ know how to send measurements to the leaders of these cycles (perhaps through multi-hop communication).

\section{A. Jacobi-Based Cycle Space Estimation}

The Jacobi-Based Cycle Space Estimation (JBCSE) algorithm is a distributed algorithm that computes optimal tension estimates. JBCSE starts with each cycle leader collecting all measurements for its cycle. Each cycle leader then computes the total discrepancy in its cycle by adding the measurements of the cycle: $\Delta_{i}=\sum_{\ell=\gamma_{i}} \tilde{z}_{\ell}$. Note that all of the discrepancies can be represented in one equation as $\Delta=C^{\prime} \tilde{z}$.

Each cycle leader possesses an iteration variable $\hat{y}_{i}$ that is initialized to zero. Let $\kappa_{i}$ be the set of cycles that share at least one edge with cycle $i$. Then, as the JBCSE algorithm proceeds, at each step each cycle leader:

1) Sends the value of its iteration variable, $\hat{y}_{i}$, to the leaders of the cycles that share edges with its own cycle, as well as to all the nodes in its cycle

2) Receives iteration variables, $\hat{y}_{\kappa_{i}}$ from the leaders of neighboring cycles

3) Updates its iteration variable,

$$
\hat{y}_{i}^{t+1}=D_{i}^{-1}\left(\sum_{\ell=\kappa_{i}} A_{i \ell} \hat{y}_{\ell}^{t}-\Delta_{i}\right) .
$$

In addition to the iteration above carried out by the cycle leaders, each node also maintains internal estimates of the optimal tensions for all its incident edges. The estimate of the optimal tension $\hat{z}_{j}^{t}$ for edge $j$ is updated as follows:

$$
\hat{z}_{j}^{t}=\tilde{z}_{j}+P_{j} \sum_{\ell=\Gamma_{j}} \hat{y}_{\ell}^{t}
$$

where $\tilde{z}_{j}$ is the measurement corresponding to the edge $j, P_{j}$ the error covariance for that edge, and $\Gamma_{j}$ denotes the set of cycles that contain the edge $j$. The set $\Gamma_{j}$ can be empty if the edge does not belong to any cycle, in which case $\hat{z}_{j}^{t}=\tilde{z}_{j}$.

To obtain its current state estimate, a node needs only to add the tensions along a path between itself and a reference node. This can be accomplished via a flagging method similar to that introduced in [1]. In this way, all non-reference nodes would start with a flagged state value, $\hat{x}^{0}=\infty$. The first time that a node, say $\mathcal{V}_{A}$, communicates with a neighbor, say $\mathcal{V}_{B}$, that has a non-flagged state, $\mathcal{V}_{A}$ will know that $\mathcal{V}_{B}$ is the end node in a path connected to a reference node. $\mathcal{V}_{A}$ will then update its state, $\hat{x}_{A}^{t+1}$, to $\mathcal{V}_{B}$ 's state, $\hat{x}_{B}^{t}$, plus or minus (depending on the orientation of the edge that connects the nodes, $\mathcal{E}_{A B}$ ) the tension estimate connecting the two nodes, $\hat{z}_{A B}^{t}$. As the state estimates, $\hat{x}^{t}$, and tension estimates, $\hat{z}^{t}$, are refined, $\mathcal{V}_{A}$ will maintain its estimate as the current state estimate for $\mathcal{V}_{B}, \hat{x}_{B}^{t}$, plus or minus the current tension estimate for the edge connecting the two nodes, $\hat{z}_{A B}^{t}$. Mathematically, this process can be expressed as:

$$
\hat{x}_{A}^{t+1} \equiv \begin{cases}\infty & \text { if } \hat{x}_{B}^{t}=\infty \\ \hat{x}_{B}^{t}+\hat{z}_{A B}^{t} & \text { if } \mathcal{E}_{A B} \text { leaves } \mathcal{V}_{A} \& \hat{x}_{B}^{t} \neq \infty \\ \hat{x}_{B}^{t}-\hat{z}_{A B}^{t} & \text { if } \mathcal{E}_{A B} \text { enters } \mathcal{V}_{A} \& \hat{x}_{B}^{t} \neq \infty\end{cases}
$$

Using this flagging method, a tree (or trees) will grow out from a reference node (or reference nodes). Under the assumption that the measurement graph is weakly connected and neighbors in the graph can communicate, the number of iterations for the flagged initialization process to complete and all nodes to have a state estimate is less than or equal to the diameter of the graph.

For a given graph, there can be many trees rooted at the reference nodes. Choosing different trees will give different state estimates, $\hat{x}$. Because a set of tensions is consistent, the sum of tensions along any path connecting two nodes will be the same. As the optimal tension estimates converge, the state estimates obtained using any tree will converge to the optimal minimum variance estimate, $\hat{x}^{*}$.

When the iteration variables are stacked into a single vector, $\hat{y}$, the update law (11) can be rewritten:

$$
\hat{y}^{t+1}=D^{-1}\left(A \hat{y}^{t}-\Delta\right) .
$$

When the tension estimates are considered in a single vector, $\hat{z}$, the update law corresponding to (12) is:

$$
\hat{z}^{t}=\tilde{z}+P C \hat{y}^{t} .
$$

Once the flagging process has finished and the state estimates, $\hat{x}$, are being updated along a tree, the state update process (13) can be represented using a single equation. Take $A_{T}$ and $B_{T}$ to be the adjacency and the incidence matrices, respectively, for a directed tree (trees) rooted at a reference node (nodes). The state update law is:

$$
\hat{x}^{t+1}=A_{T} \hat{x}^{t}+B_{T} \hat{z}^{t} .
$$

Equations (14), (15), and (16) can be combined into the following linear equation representing the JBCSE algorithm:

$$
\left[\begin{array}{l}
\hat{y} \\
\hat{x}
\end{array}\right]^{+}=\left[\begin{array}{cc}
D^{-1} A & 0 \\
B_{T} P C & A_{T}
\end{array}\right]\left[\begin{array}{l}
\hat{y} \\
\hat{x}
\end{array}\right]-\left[\begin{array}{c}
D^{-1} C^{\prime} \\
-B_{T}
\end{array}\right] \tilde{z} .
$$


Theorem 3: [Sufficient Condition for Convergence] Given the cycle Laplacian of a graph, $\mathcal{L}_{c}=D-A$, where $D$ and $A$ are the weighted cycle degree matrix and the weighted cycle adjacency matrix, respectively, if the spectral radius of $D^{-1} A$ is strictly less than 1 , then $\hat{z}^{t}$ in (15) converges to the optimal tension estimate in (4).

Proof: From (14), when $\hat{y}^{t}$ converges, $\hat{y}^{*}=$ $D^{-1}\left(A \hat{y}^{*}-\Delta\right)$. The error between $\hat{y}^{t}$ and $\hat{y}^{*}$ has the following dynamics:

$$
\begin{aligned}
\hat{y}^{t+1}-\hat{y}^{*} & =D^{-1}\left(A \hat{y}^{t}-\Delta\right)-D^{-1}\left(A \hat{y}^{*}-\Delta\right) \\
& =D^{-1} A\left(\hat{y}^{t}-\hat{y}^{*}\right) .
\end{aligned}
$$

When the spectral radius of $D^{-1} A$ is strictly less than 1 , the error between $\hat{y}^{t}$ and $\hat{y}^{*}$ goes to zero as $t$ goes to infinity. Similarly, from (15), when $\hat{z}^{t}$ converges, $\hat{z}^{*}=\tilde{z}+P C \hat{y}^{*}$. The error between $\hat{z}^{t}$ and $\hat{z}^{*}$ has the following dynamics:

$$
\begin{aligned}
\hat{z}^{t+1}-\hat{z}^{*} & =\tilde{z}+P C \hat{y}^{t}-\tilde{z}+P C \hat{y}^{*} \\
& =P C\left(\hat{y}^{t}-\hat{y}^{*}\right) .
\end{aligned}
$$

If the spectral radius of $D^{-1} A$ is less than 1 and $\hat{y}^{t}-\hat{y}^{*}$ goes to zero as $t$ goes to infinity, then $\hat{z}^{t}-\hat{z}^{*}$ will also go to zero as $t$ goes to infinity. Furthermore, when $\hat{y}^{t}$ converges:

$$
\begin{aligned}
\hat{y}^{*} & =D^{-1}\left(A \hat{y}^{*}-\Delta\right) \\
D \hat{y}^{*} & =A \hat{y}^{*}-\Delta \\
(D-A) \hat{y}^{*} & =-\Delta \\
\left(C^{\prime} P C\right) \hat{y}^{*} & =-C^{\prime} \tilde{z} \\
\hat{y}^{*} & =-\left(C^{\prime} P C\right)^{-1} C^{\prime} \tilde{z},
\end{aligned}
$$

from which, $\hat{z}^{*}=\tilde{z}-P C\left(C^{\prime} P C\right)^{-1} C^{\prime} \tilde{z}$, as in (4).

When a planar graph is drawn such that no edges cross, the plane is divided into contiguous regions called faces (see Figure 1 for an example of graph faces). A graph face is characterized by the cycle that forms its boundary [6]. The faces of the graph are taken to be the set of all faces in the graph. The following theorem provides a convergence result for planar graphs. In this theorem, the faces of the graph are chosen as a basis for the cycle space.

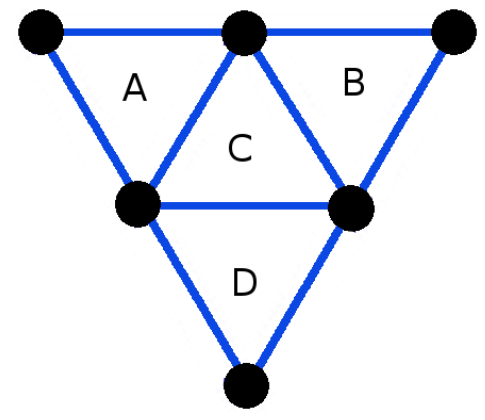

Fig. 1. A simple graph with faces $\mathrm{A}, \mathrm{B}, \mathrm{C}$, and D.
Theorem 4: [Convergence for Planar Graphs]

When $\mathcal{G}$ is a planar graph and $C$ is the basis of the cycle space that coincides with the faces of the graph, then the discrete time linear system in (17) converges and $\lim _{t \rightarrow \infty} \hat{x}^{t}=\hat{x}^{*}$, the BLUE of $x$.

Proof: For any planar graph when the cycle space is chosen to be the faces of the graph, then $D_{i i}$ is the sum of edge weightings around cycle $i$ and $\left|A_{i j}\right|$ is the sum of edge weightings for edges in both cycle $i$ and cycle $j$ (with $A_{i i}$ defined to be zero). Because the cycle space is defined to be the the faces of a planar graph, an edge exists in at most two cycles hence $D_{i i} \geq \sum_{j=1}^{d n} A_{i j}$. For all cycles on the outside of the graph, the inequality is strict because these cycles have at least one edge not shared with another cycle. Hence $\mathcal{L}_{c}$ is weakly diagonally dominant. Furthermore, when all of the cycles are connected (cycles are connected if they share an edge), $\mathcal{L}_{c}$ is irreducible. Because $\mathcal{L}_{c}$ is weakly diagonally dominant and irreducible, the iteration algorithm converges. In the planar graph case, the irreducibility condition can be relaxed because every cluster of connected cycles contains at least one cycle with a non-shared edge. Hence, each group of connected cycles has at least one cycle with strong dominance and the iteration algorithm converges.

\section{Convergence Rate of the JBCSE Algorithm}

The rate of convergence for the Jacobi iteration algorithm, $\rho(J)$, is the spectral radius of the iteration matrix, $J=$ $D^{-1} A$. Currently, no closed form solution exists to find $\rho(J)$ for a general measurement graph. However, a closed form expression for $\rho(J)$ does exist for the JBCSE algorithm applied to a square lattice of measurements with uniform error covariances.

\section{Theorem 5: [Convergence Rate of JBCSE on a Grid]}

Consider a $K$ x $L$ grid of nodes connected by measurements with uniform covariances. The convergence rate of the JBCSE algorithm is:

$$
\rho\left(\mathcal{L}_{c}\right)=\frac{1}{2}\left[\cos \left(\frac{\pi}{K}\right)+\cos \left(\frac{\pi}{L}\right)\right] .
$$

Proof: Start by labeling the nodes with grid coordinates: $\mathcal{V}=v_{\bar{k}, \bar{l}}$, where $\bar{k} \in\{1,2, \ldots, K\}$ and $\bar{l} \in$ $\{1,2, \ldots, L\}$. Let $C$ be the set of cycles corresponding to the faces of the graph, the cycle space is a $(K-1) \mathrm{x}$ $(L-1)$ grid: $C=c_{k, l}$ where $k \in\{1,2, \ldots, K-1\}$ and $l \in\{1,2, \ldots, L-1\}$.

Because the cycle interconnections form of a grid, when the iteration variables, $\hat{y}_{k, l}$, for $k \in\{1,2, \ldots, K-1\}$ and $l \in\{1,2, \ldots, L-1\}$, are updated as per (11):

$$
\hat{y}_{k, l}^{+}=\frac{1}{4}\left(\hat{y}_{k+1, l}+\hat{y}_{k+1, l}+\hat{y}_{k, l+1}+\hat{y}_{k, l-1}\right)-\frac{1}{4} \Delta_{k, l},
$$

where $y_{0, l}=y_{K, l}=y_{k, 0}=y_{k, L}=0$. In steady state:

$$
\hat{y}_{k, l}^{*}=\frac{1}{4}\left(\hat{y}_{k+1, l}^{*}+\hat{y}_{k+1, l}^{*}+\hat{y}_{k, l+1}^{*}+\hat{y}_{k, l-1}^{*}\right)-\frac{1}{4} \Delta_{k, l} .
$$


The error between $\hat{y}_{k, l}$ and $\hat{y}_{k, l}^{*}$, for $k \in\{1,2, \ldots, K-1\}$ and $l \in\{1,2, \ldots, L-1\}$, evolves as:

$$
\hat{e}_{k, l}^{+}=\frac{1}{4}\left(\hat{e}_{k+1, l}+\hat{e}_{k+1, l}+\hat{e}_{k, l+1}+\hat{e}_{k, l-1}\right)
$$

where $e_{0, l}=e_{K, l}=e_{k, 0}=e_{k, L}=0$. Equation (18) is a Discrete Poisson Equation with homogeneous Dirichlet boundary conditions. From [3], a Discrete Poisson Equation with homogeneous Dirichlet boundary conditions have eigenfunctions of the form:

$$
\hat{e}_{k, l}=\sin \left(\frac{\pi \mu k}{K}\right) \sin \left(\frac{\pi \nu l}{L}\right)
$$

where $\mu \in\{1,2, \ldots, K-1\}$ and $\nu \in\{1,2, \ldots, L-1\}$. Eigenvalues for (18) can be found by substituting (19):

$$
\lambda \hat{e}_{k, l}=\frac{1}{4}\left(\hat{e}_{k+1, l}+\hat{e}_{k+1, l}+\hat{e}_{k, l+1}+\hat{e}_{k, l-1}\right),
$$

as such:

$$
\begin{aligned}
& \lambda\left[\sin \left(\frac{\pi \mu k}{K}\right)\right.\left.\sin \left(\frac{\pi \nu l}{L}\right)\right]= \\
& \frac{1}{4}\left[\sin \left(\frac{\pi \mu(k+1)}{K}\right) \sin \left(\frac{\pi \nu l}{L}\right)+\right. \\
& \quad \sin \left(\frac{\pi \mu(k-1)}{K}\right) \sin \left(\frac{\pi \nu l}{L}\right)+ \\
& \sin \left(\frac{\pi \mu k}{K}\right) \sin \left(\frac{\pi \nu(l+1)}{L}\right)+ \\
&\left.\sin \left(\frac{\pi \mu k}{K}\right) \sin \left(\frac{\pi \nu(l-1)}{L}\right)\right] .
\end{aligned}
$$

Making use of the trigonometric identity, $\sin (a+b)=$ $\sin (a) \cos (b)+\cos (a) \sin (b),(20)$ reduces to:

$$
\begin{aligned}
& \lambda\left[\sin \left(\frac{\pi \mu k}{K}\right)\right.\left.\sin \left(\frac{\pi \nu l}{L}\right)\right]= \\
& \frac{1}{2}\left[\sin \left(\frac{\pi \mu k}{K}\right) \sin \left(\frac{\pi \nu l}{L}\right) \cos \left(\frac{\pi \mu}{K}\right)+\right. \\
&\left.\quad \sin \left(\frac{\pi \mu k}{K}\right) \sin \left(\frac{\pi \nu l}{L}\right) \cos \left(\frac{\pi \nu}{L}\right)\right],
\end{aligned}
$$

which simplifies further to:

$$
\lambda=\frac{1}{2}\left[\cos \left(\frac{\pi \mu}{K}\right)+\cos \left(\frac{\pi \nu}{L}\right)\right],
$$

which is largest in magnitude when $\mu=1$ and $\nu=1$. Hence, the spectral radius of linear system (18) is

$$
\lambda_{\max }=\frac{1}{2}\left[\cos \left(\frac{\pi}{K}\right)+\cos \left(\frac{\pi}{L}\right)\right] .
$$

\section{Simulations}

This section presents a comparison between the Jacobi Algorithm with flagged initialization (JAWFI) introduced in [1] and the JBCSE algorithm. While it is not practical to compare performance on every type of graph, this section does explore performance for a wide range of regular graphs that include triangle, square, and hexagonal lattices. The simulation results demonstrate the performance gains possible when using JBCSE.

The simulations were run on lattices of various size. The nodes in each lattice are one unit away from their edge neighbors. Measurements are corrupted by zero mean Gaussian noise with standard deviation 0.25 . For each set of measurements, both algorithms were run until the 2-norm of estimation error normalized by the number of nodes, $\frac{\left\|\hat{x}^{*}-\hat{x}\right\|}{n}$, was below 0.025 (one tenth of the standard deviation of the measurement noise). This process was repeated on six hundred sets of measurements for each size lattice graph. The size of a lattice was taken to be the number of tessellations on one side of a given lattice. Figure 2 shows the first three sizes for each type of lattice.

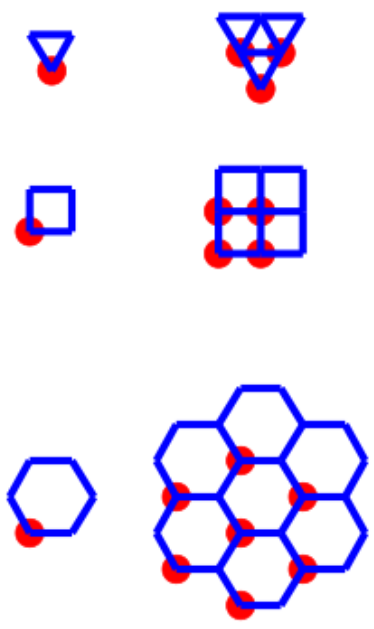

Fig. 2. Size one, two, and three lattices with cycle leaders highlighted.

Figure 3 depicts the ratio of the average number of iterations needed by the JBCSE over the average number of iterations needed by for the JAWFI method, as a function of the lattice size. Significant gains in terms of the number of iterations can be had for large graphs. Figure 4 charts the ratio of the average number of messages passed by the JBCSE algorithm over the average number of messages passed by the JAWFI method, as a function of lattice size. For the JBCSE algorithm, messages passed during computation of cycle discrepancies, during the flagged initialization process, between cycle leaders during the iteration process, and from cycle leaders to other nodes for state updates were all counted. In both of these figures, the horizontal axis corresponds to the size of the lattice. For smaller lattices, the overhead of gathering measurements makes JBCSE less 


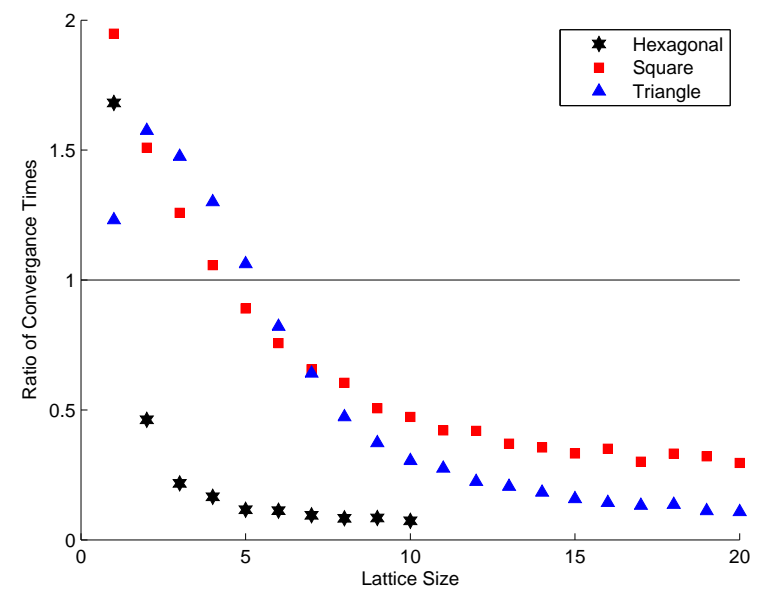

Fig. 3. The average number of iterations for JBCSE to finish over the average number of iterations for JAWFI to finish.

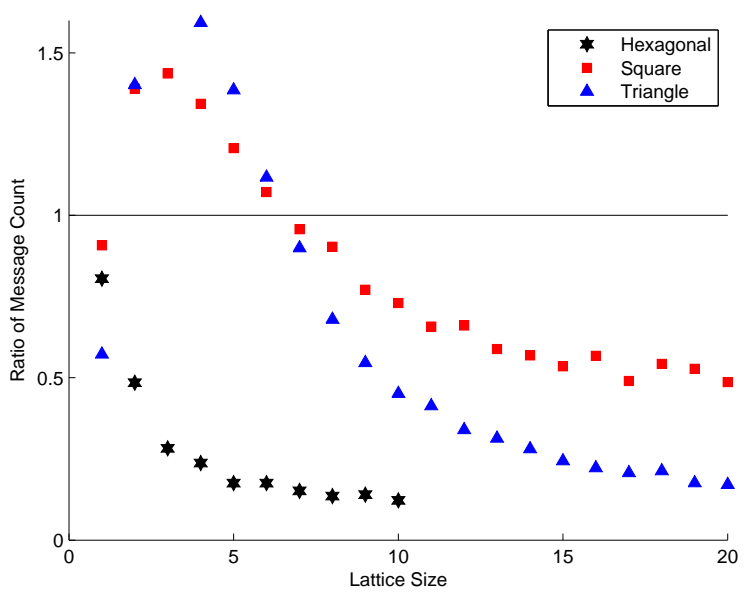

Fig. 4. The average number of messages passed during JBCSE over the average number of messages passed during JAWFI.

advantageous, but for larger graphs, JBCSE shows faster convergence with less message passing. In both Figure 3 and Figure 4, results for hexagonal lattice graphs stop at size ten due to lengthy JAWFI convergence times.

\section{CONCLUSION}

This paper introduced cycle space estimation from relative measurements and showed its equivalence to BLUE. It introduced a Jacobi based distributed algorithm that was shown to converge (under appropriate conditions) to the BLUE. Sufficient conditions for convergence of the distributed algorithm were given, as well as a closed form convergence rates for the algorithm on a grid network.

To make the methods in this paper more attractive, a few items need to be explored further. In order to get the most distributed JBCSE, one must be able to determine the faces of a network in a distributed manner. It is also interesting to study how the selection of the cycle leader nodes in the graph affects performance of distributed cycle space algorithms.

\section{APPENDIX}

Lemma 1: Let $\alpha$ and $\beta$ be linear subspaces where $\operatorname{null}(\operatorname{proj}(\alpha))=\beta$. Given that $\alpha\left(\alpha^{\prime} \alpha\right)^{-1} \alpha^{\prime}$ is a projection on $\alpha^{\prime}$ and $\beta^{\prime}\left(\beta \beta^{\prime}\right)^{-1} \beta$ is a projection on $\beta$, the following equality holds:

$$
\alpha\left(\alpha^{\prime} \alpha\right)^{-1} \alpha^{\prime}=I-\beta^{\prime}\left(\beta \beta^{\prime}\right)^{-1} \beta
$$

Proof:

$$
\operatorname{null}(\operatorname{proj}(\alpha))=\beta \quad \Rightarrow \quad \beta \perp \alpha^{\prime}
$$

Because $\alpha^{\prime}$ and $\beta$ are orthogonal complements, so are their projection matrices:

$$
\left(\beta^{\prime}\left(\beta \beta^{\prime}\right)^{-1} \beta\right)^{\perp}=\alpha\left(\alpha^{\prime} \alpha\right)^{-1} \alpha^{\prime}
$$

As demonstrated in [10], a matrix projection, $\mathcal{P}$, is an orthogonal complement to $I-\mathcal{P}$. This implies:

$$
\left(\beta^{\prime}\left(\beta \beta^{\prime}\right)^{-1} \beta\right)^{\perp}=I-\beta^{\prime}\left(\beta \beta^{\prime}\right)^{-1} \beta
$$

From equations (VIII) and (VIII):

$$
\alpha\left(\alpha^{\prime} \alpha\right)^{-1} \alpha^{\prime}=I-\beta^{\prime}\left(\beta \beta^{\prime}\right)^{-1} \beta
$$

\section{ACKNOWLEDGEMENTS}

Thank you to Saul Teukolsky for personal correspondence outlining the proof of Theorem 5 .

\section{REFERENCES}

[1] P. Barooah, N.M. da Silva, and J.P. Hespanha. Distributed optimal estimation from relative measurements for localization and time synchronization. In P.B. Gibbons, T. Abdelzaher, J. Aspnes, and R. Rao, editors, Distributed Computing in Sensor Systems, volume 4026, pages 266-281. Springer, Berlin, June 2006. Presented at the Int. Conf. on Distributed Computing in Sensor Systems (DCOSS): Algorithms track.

[2] H. Chen, T. Kirubarajan, and Y. Bar-Shalom. Performance limits of track-to-track fusion versus centralized estimation: theory and application [sensor fusion]. IEEE Transactions on Aerospace and Electronic Systems, 39(2):386-400, 2003.

[3] D. Dubin. Numerical and Analytical Methods for Scientists and Engineers Using Mathematica. Wiley-Interscience, Hoboken, NJ, 2003.

[4] J.A. Fax and R.M. Murray. Information flow and cooperative control of vehicle formations. IEEE Transactions on Automatic Control, 49(9):1465-1476, 2004.

[5] F. Gustafsson and F. Gunnarsson. Positioning using time-difference of arrival measurements. In Proceedings of ICASSP, 2003.

[6] R. Johnsonbaugh. Discrete Mathematics. Pearson Prentice Hall, Upper Saddle River, NJ, 2009.

[7] J.M. Ortega. Numerical Analysis A Second Course. Society for Industrial and Applied Mathematics, Philadelphia, PA, 1990.

[8] R.T. Rockafellar. Network Flows and Monotropic Optimization. Athena Scientific, Nashua, NH, 1998.

[9] R. Solis, V.S. Borkar, and P.R. Kumar. A new distributed time synchronization protocol for multihop wireless networks. In Decision and Control, 2006 45th IEEE Conference on, pages 2734-2739, 2006.

[10] E.S. Suhubi. Functional Analysis. Kluwer Academic Publishers, Norwell, MA, 1990. 